Proyecciones Journal of Mathematics

Vol. 29, No 2, pp. 83-92, August 2010.

Universidad Católica del Norte

Antofagasta - Chile

\title{
INFORMATION MATRIX FOR GENERALIZED SKEW - NORMAL DISTRIBUTIONS
}

\author{
HÉCTOR W. GÓMEZ \\ UNIVERSIDAD DE ANTOFAGASTA, CHILE \\ and \\ HUGO S. SALINAS \\ UNIVERSIDAD DE ATACAMA, CHILE \\ Received: April 2009. Accepted: March 2010
}

\begin{abstract}
The Fisher information matrix for Generalized skew-normal (GSN) distribution is derived. The expressions for the elements of the matrices require of integrals that are solved numerically using a suitable software.
\end{abstract}

Key words: Generalized skew-normal distribution; Skew-normal distribution; Normal distribution 


\section{Introduction}

Consider the generalized skew-normal distribution (GSN) introduced by Gómez et al. [4], whose pdf is given by

$$
f(x)= \begin{cases}\frac{2}{\sigma} \phi\left(\frac{z}{1+\beta}\right)\left[\frac{\beta}{1+\beta}+\frac{(1-\beta)}{1+\beta} \Phi\left(\frac{\lambda z}{1+\beta}\right)\right], & \text { if } x<\mu, \\ \frac{2}{\sigma} \phi\left(\frac{z}{1-\beta}\right) \Phi\left(\frac{\lambda z}{1-\beta}\right), & \text { if } x \geq \mu,\end{cases}
$$

where $z=(x-\mu) / \sigma, \mu \in R, \sigma>0, \lambda \in R, \beta \in[0,1)$, and $\phi($.$) and \Phi($. denote the pdf and cdf of the standardized normal distribution, respectively. The main properties and the convenience of this model to fit asymmetric data are discussed in Gómez et al. [4].

The main objective of this note is to calculate the Fisher information matrix corresponding to (1.1), which plays an important role in the asymptotic variance of the maximum likelihood estimators [5] and is defined in the following manner: Let $\boldsymbol{X}=\left(X_{1}, \ldots, X_{n}\right)$ be a random sample, and let $f(\boldsymbol{X} ; \boldsymbol{\theta})$ denote the probability density function for some model of the data, which has parameter vector $\boldsymbol{\theta}=\left(\theta_{1}, \ldots, \theta_{p}\right)$. Then the Fisher information matrix $I_{n}(\boldsymbol{\theta})$ of sample size $n$ is given by the $p \times p$ symmetric matrix whose $i j$-th element is given by the covariance between first partial derivatives of the log-likelihood,

$$
I_{n}(\boldsymbol{\theta})_{i j}=\operatorname{Cov}\left[\frac{\partial \log f(\boldsymbol{X} ; \boldsymbol{\theta})}{\partial \theta_{i}}, \frac{\partial \log f(\boldsymbol{X} ; \boldsymbol{\theta})}{\partial \theta_{j}}\right] .
$$

An alternative, but equivalent, definition for the Fisher information matrix is based on the expected values of the second partial derivatives, and is given by

$$
I_{n}(\boldsymbol{\theta})_{i j}=-E\left[\frac{\partial^{2} \log f(\boldsymbol{X} ; \boldsymbol{\theta})}{\partial \theta_{i} \partial \theta_{j}}\right]
$$

Strictly, this definition corresponds to the expected Fisher information. If no expectation is taken we obtain a data-dependent quantity that is called the observed Fisher information.

The rest of this note is organized as follows. The elements of the expected information matrix for the full location-scale GSN model are derived in Section 2. Sections 3 analyze the special case of the sub-models skewnormal and normal. For the normal case, the information matrix is singular, and a reparametrization obtained by using the iterative approach proposed by Rotnitzky et al. [7] is used to solve this problem, and a final discussion is given in Section 4. The technical details are given in an appendix. 


\section{Maximum likelihood estimation}

This section is related to the asymptotic properties of the MLEs of the location-scale GSN model. Specifically, the ingredients to compute the expected information matrix for the full location-scale GSN model are given. Hence, the study is focused on the asymptotic behavior of the MLEs for the particular skew-normal and normal models.

\subsection{Likelihood score functions}

Let $X_{1}, \ldots, X_{n}$ be a random sample drawn from the $\operatorname{GSN}(\mu, \sigma, \lambda, \beta)$ distribution. The log- likelihood function for $\theta=(\mu, \sigma, \lambda, \beta)^{\top}$ is $\sum_{i=1}^{n} l\left(\theta, X_{i}\right)$, where $l(\theta, X)$ is the log-likelihood for $\theta$ based on a single observation $X$, that is,

$$
\begin{aligned}
& l(\theta ; X):=\log f(X ; \theta) \\
& =\log \left(\frac{2}{\sigma}\right)+\log \phi\left(\frac{Z}{1+\beta}\right) I_{A}(X)+\log \phi\left(\frac{Z}{1-\beta}\right) I_{A^{\prime}}(X) \\
& +\log \left[\frac{\beta}{1+\beta}+\frac{(1-\beta)}{1+\beta} \Phi\left(\frac{\lambda Z}{1+\beta}\right)\right] I_{A}(X)+\log \Phi\left(\frac{\lambda Z}{1-\beta}\right) I_{A^{\prime}}(X),
\end{aligned}
$$

where $Z=(X-\mu) / \sigma, A=\{X \mid X<\mu\}, A^{\prime}$ is the complement of set $A$ and $I_{A}$ is the indicator function of set $A$. The score function is $\sum_{i=1}^{n} S_{\theta}\left(\theta, X_{i}\right)$, where $S_{\theta}(\theta, X)=\partial l(\theta, X) / \partial \theta$ is the vector $\left(S_{\mu}, S_{\sigma}, S_{\lambda}, S_{\beta}\right)^{\top}$ with elements

$\mathrm{S}_{\mu}=\left[\frac{Z}{\sigma(1+\beta)^{2}}-\frac{\lambda(1-\beta) S(Z)}{\sigma(1+\beta)}\right] I_{A}(X)+\left[\frac{Z}{\sigma(1-\beta)^{2}}-\frac{\lambda R(Z)}{\sigma(1-\beta)}\right] I_{A^{\prime}}(X)$,

$S_{\sigma}=-1 / \sigma+\left[\frac{Z^{2}}{\sigma(1+\beta)^{2}}-\frac{\lambda(1-\beta) Z S(Z)}{\sigma(1+\beta)}\right] I_{A}(X)+\left[\frac{Z^{2}}{\sigma(1-\beta)^{2}}-\frac{\lambda Z R(Z)}{\sigma(1-\beta)}\right] I_{A^{\prime}}(X)$,

$S_{\lambda}=\frac{(1-\beta) Z S(Z)}{1+\beta} I_{A}(X)+\frac{Z R(Z)}{1-\beta} I_{A^{\prime}}(X)$,

and

$$
\begin{aligned}
S_{\beta} & =\left[\frac{Z^{2}}{(1+\beta)^{3}}+T(Z)-\frac{\lambda(1-\beta) Z S(Z)}{(1+\beta)^{2}}-\frac{1}{1+\beta}\right] I_{A}(X) \\
& +\left[-\frac{Z^{2}}{(1-\beta)^{3}}+\frac{\lambda Z R(Z)}{(1-\beta)^{2}}\right] I_{A^{\prime}}(X)
\end{aligned}
$$

where $R(Z)=\phi\left(\frac{\lambda Z}{1-\beta}\right) / \Phi\left(\frac{\lambda Z}{1-\beta}\right), S(Z)=\phi\left(\frac{\lambda Z}{1+\beta}\right) /\left\{\beta+(1-\beta) \Phi\left(\frac{\lambda Z}{1+\beta}\right)\right\}$ 
and $T(Z)=\left\{1-\Phi\left(\frac{\lambda Z}{1+\beta}\right)\right\} /\left\{\beta+(1-\beta) \Phi\left(\frac{\lambda Z}{1+\beta}\right)\right\}$

\subsection{Fisher information matrix}

By definition, the GSN-expected information matrix for $\theta$ can be computed as (1.3). Thus, the elements $I_{\theta_{i} \theta_{j}}=E\left[-\frac{\partial^{2} l(\theta ; X)}{\partial \theta_{i} \partial \theta_{j}}\right]$ of this matrix are shown in the Appendix to be

$$
\begin{aligned}
& I_{\mu \mu}=\frac{\pi(1+\beta)+4 \lambda \beta \delta^{2}+4 \beta \arctan (\lambda)}{\sigma^{2}(1-\beta)(1+\beta)^{2} \pi}+\frac{\lambda^{2}(1-\beta)^{2}}{\sigma^{2}(1+\beta)^{2}} \rho_{0}+\frac{\lambda^{2}}{\sigma^{2}(1-\beta)^{2}} \eta_{0}, \\
& I_{\mu \sigma}=\frac{b \delta\left(1+\delta^{2}\right)}{\sigma^{2}(1+\beta)}+\frac{\lambda^{2}(1-\beta)^{2}}{\sigma^{2}(1+\beta)^{2}} \rho_{1}+\frac{\lambda^{2}}{\sigma^{2}(1-\beta)^{2}} \eta_{1} \\
& I_{\mu \lambda}=\frac{b \delta\left(1-\delta^{2}\right)}{\sigma \lambda(1+\beta)}-\frac{\lambda(1-\beta)^{2}}{\sigma(1+\beta)^{2}} \rho_{1}-\frac{\lambda}{\sigma(1-\beta)^{2}} \eta_{1}, \\
& I_{\mu \beta}=\frac{b\left(\beta^{2} \delta-4 \beta \delta-4 \beta \delta^{3}-\delta-4 \beta-4\right)}{2 \sigma(1+\beta)^{2}(1-\beta)}+\frac{\lambda^{2}(1-\beta)^{2}}{\sigma(1+\beta)^{3}} \rho_{1}-\frac{\lambda^{2}}{\sigma(1-\beta)^{3}} \eta_{1}-\frac{\lambda(1-\beta)}{\sigma(1+\beta)} \kappa_{0}, \\
& I_{\sigma \sigma}=\frac{2}{\sigma^{2}}+\frac{\lambda^{2}(1-\beta)^{2}}{\sigma^{2}(1+\beta)^{2}} \rho_{2}+\frac{\lambda^{2}}{\sigma^{2}(1-\beta)^{2}} \eta_{2}, \\
& I_{\sigma \lambda}=-\frac{\lambda(1-\beta)^{2}}{\sigma(1+\beta)^{2}} \rho_{2}-\frac{\lambda}{\sigma(1-\beta)^{2}} \eta_{2}, \\
& I_{\sigma \beta}=\frac{\delta^{2}\left(\beta-4 \delta^{2}-1\right)-4 \lambda \arctan (\lambda)}{\sigma(1+\beta) \lambda \pi}+\frac{\lambda^{2}(1-\beta)^{2}}{\sigma(1+\beta)^{3}} \rho_{2}-\frac{\lambda^{2}}{\sigma(1-\beta)^{3}} \eta_{2}-\frac{\lambda(1-\beta)}{\sigma(1+\beta)} \kappa_{1}, \\
& I_{\lambda \lambda}=\frac{(1-\beta)^{2}}{(1+\beta)^{2}} \rho_{2}+\frac{1}{(1-\beta)^{2}} \eta_{2}, \\
& I_{\lambda \beta}=\frac{4 \delta^{4}-(3+\beta) \delta^{2}}{\lambda^{2}(1+\beta) \pi}-\frac{\lambda(1-\beta)^{2}}{(1+\beta)^{3}} \rho_{2}+\frac{\lambda}{(1-\beta)^{3}} \eta_{2}+\frac{1-\beta}{1+\beta} \kappa_{1}, \\
& I_{(1+\beta)}
\end{aligned}
$$

and

$$
\begin{aligned}
& I_{\beta \beta}=\frac{(1+\beta)\left\{\pi \lambda(5+\beta)+4 \beta \delta^{4}(3-\beta)\right\}+4 \delta^{2}\left(1+2 \beta-\beta^{2}\right)+24 \beta \lambda \arctan (\lambda)}{2 \pi \lambda(1-\beta)(1+\beta)^{2}} \\
& +\frac{\lambda^{2}(1-\beta)^{2}}{(1+\beta)^{3}} \rho_{2}+\frac{\lambda^{2}}{(1-\beta)^{4}} \eta_{2}-\frac{\lambda(1-\beta)(2+\beta)}{(1+\beta)^{2}} \kappa_{1}+v_{0}
\end{aligned}
$$


where $b=\sqrt{2 / \pi}$ and $\delta=\lambda / \sqrt{1+\lambda^{2}}$. The factors $\rho_{k}, \eta_{k}, \kappa_{k}$ and $v_{k}$ are defined in the Appendix.

\section{Special Cases}

When $\beta=0$, with $\lambda \neq 0$, (1.1) reduces to the skew-normal (SN) pdf introduced by Azzalini [1]. For this particular case, the elements of the Fisher information matrix are

$I_{\mu \mu}=\frac{1}{\sigma^{2}}+\frac{\lambda^{2}}{\sigma^{2}} a_{0}, \quad I_{\mu \sigma}=\frac{b \delta\left(1+\delta^{2}\right)}{\sigma^{2}}+\frac{\lambda^{2}}{\sigma^{2}} a_{1}, \quad I_{\mu \lambda}=\frac{b \delta\left(1-\delta^{2}\right)}{\sigma \lambda}-\frac{\lambda}{\sigma} a_{1}$,

$I_{\mu \beta}=-\frac{b(\delta+4)}{2 \sigma}+\frac{\lambda^{2}}{\sigma}\left(\rho_{1}^{*}-\eta_{1}^{*}\right)-\frac{\lambda}{\sigma} \kappa_{0}^{*}, \quad I_{\sigma \sigma}=\frac{2}{\sigma^{2}}+\frac{\lambda^{2}}{\sigma^{2}} a_{2}, \quad I_{\sigma \lambda}=-\frac{\lambda}{\sigma} a_{2}$,

$I_{\sigma \beta}=-\frac{\delta^{2}\left(4 \delta^{2}+1\right)+4 \lambda \arctan (\lambda)}{\sigma \lambda \pi}+\frac{\lambda^{2}}{\sigma}\left(\rho_{2}^{*}-\eta_{2}^{*}\right)-\frac{\lambda}{\sigma} \kappa_{1}^{*}, \quad I_{\lambda \lambda}=a_{2}$,

$I_{\lambda \beta}=\frac{\delta^{2}\left(4 \delta^{2}-3\right)}{\lambda^{2} \pi}-\lambda\left(\rho_{2}^{*}-\eta_{2}^{*}\right)+\kappa_{1}^{*}, \quad$ and $\quad I_{\beta \beta}=\frac{5 \pi \lambda+4 \delta^{2}}{2 \pi \lambda}+\lambda^{2} a_{2}-2 \lambda \kappa_{1}^{*}+v_{0}^{*}$,

where $a_{k}=E_{S N}\left[Z^{k}\left\{\frac{\phi(\lambda Z)}{\Phi(\lambda Z)}\right\}^{2}\right]$. The coefficients $\rho_{k}^{*}, \eta_{k}^{*}, \kappa_{k}^{*}$ and $v_{k}^{*}$ are given in the Appendix.

Note the submatrix corresponding to the parameters $\mu, \sigma$ y $\lambda$ coincides with the result obtained by Azzalini [1]. Note also that when $\lambda \neq 0$, the observed information matrix is non-singular, so that it can be used also to estimate the asymptotic variances of the maximum likelihood estimators.

On the other hand, when $\beta=0$ and $\lambda=0,(1)$ reduces to the normal model. For this submodel, the Fisher information matrix is

$$
\left(\begin{array}{cccc}
\frac{1}{\sigma^{2}} & 0 & \frac{\sqrt{2}}{\sigma \sqrt{\pi}} & -\frac{2 \sqrt{2}}{\sigma \sqrt{\pi}} \\
0 & \frac{2}{\sigma^{2}} & 0 & 0 \\
\frac{\sqrt{2}}{\sigma \sqrt{\pi}} & 0 & \frac{2}{\pi} & -\frac{4}{\pi} \\
-\frac{2 \sqrt{2}}{\sigma \sqrt{\pi}} & 0 & -\frac{4}{\pi} & 3
\end{array}\right) .
$$

Note from this matrix that the column corresponding to the parameters $\mu$ are $\lambda$ are linearly depended, implying thus that it is a singular. This irregularity is discussed by Azzalini [1] in the context of the SN model, and posteriorly it is studied systematically by Chiogna [2]. DiCiccio and 
Monti [3] studying also this singularity in the context of skew-exponential power distribution, and similar to Chiogna [2], they using the methodology of Rotnitzky et al. [7] to obtain an appropriated reparametrization from which is possible to calculate the asymptotic distribution of the maximum likelihood estimator.

In the present case, this approach will also employed. For this, it is assumes that the random sample becomes from a $N\left(\mu^{*}, \sigma^{*}\right)$ model, where $\boldsymbol{\theta}^{*}=\left(\mu^{*}, \sigma^{*}, 0,0\right)$. Then, from the iterative procedure of Rotnitzky et al. [7], the following reparametrization is obtained: $\tilde{\boldsymbol{\theta}}=\tilde{\boldsymbol{\theta}}(\boldsymbol{\theta})=(\tilde{\mu}, \tilde{\sigma}, \lambda, \beta)$, where

$$
\tilde{\mu}=\mu+\sigma^{*} b \lambda, \quad \tilde{\sigma}=\sigma-\frac{1}{2} \sigma^{*} b^{2} \lambda^{2}
$$

Observe that the parameter $\boldsymbol{\theta}^{*}$ remains unchanged under the new parametrization. Finally, from the Theorem 3 in Rotnitzky et al. [7], it follows that, under $H_{0}: \boldsymbol{\theta}=\boldsymbol{\theta}^{*}$, the random vector

$$
\left[n^{1 / 2}\left(\widehat{\mu}-\mu^{*}+b \sigma^{*} \widehat{\lambda}\right), n^{1 / 2}\left(\widehat{\sigma}-\sigma^{*}-1 / 2 b^{2} \sigma^{*} \widehat{\lambda}^{2}\right), n^{1 / 6} \widehat{\lambda}, n^{1 / 2} \widehat{\beta}\right]
$$

converge in distribution to $\left(Z_{1}, Z_{2}, Z_{3}^{1 / 3}, Z_{4}\right)$, where $\left(Z_{1}, Z_{2}, Z_{3}, Z_{4}\right)$ is a normal random vector with mean zero and covariance matrix

$$
\left(\begin{array}{cccc}
\frac{1}{\sigma^{2}} & 0 & \frac{1}{\sigma} \frac{2-\pi}{\sqrt{2 \pi^{3}}} & -\frac{2 \sqrt{2}}{\sigma \sqrt{\pi}} \\
0 & \frac{2}{\sigma^{2}} & 0 & 0 \\
\frac{1}{\sigma} \frac{2-\pi}{\sqrt{2 \pi^{3}}} & 0 & \frac{5 \pi^{2}-28 \pi+44}{6 \pi^{3}} & \frac{8 \pi-20}{3 \pi^{2}} \\
-\frac{2 \sqrt{2}}{\sigma \sqrt{\pi}} & 0 & \frac{8 \pi-20}{3 \pi^{2}} & 3
\end{array}\right)^{-1}
$$

\section{A final discussion}

In this paper we study the singularity of the Fisher information matrix of the generalized skew-normal model (GSN). This problem has been studied in the context of skew-normal model and the same applies to the GSN model, i.e. the information matrix for the generalized skew-normal model is singular for $\beta=\lambda=0$. Obtain a nonsingular matrix for the generalized skew-normal model using the algorithm Rotnitzky et al. [7].

\section{Acknowledgement}

The authors acknowledge helpful comments and suggestions of the referee, which substantially improved the presentation. HW Gómez was partially 
supported by Grant FONDECYT 1090411 while HS Salinas was Partially supported by Grant DIUDA 221180.

\section{Appendix}

The second-order derivatives of $l(\theta ; X)$ are

$$
\begin{aligned}
& \frac{\partial^{2} l(\theta ; X)}{\partial \mu^{2}}=-\frac{1}{\sigma^{2}}\left[\frac{1}{(1+\beta)^{2}}+\frac{\lambda^{3}(1-\beta)}{(1+\beta)^{3}} Z S(Z)+\frac{\lambda^{2}(1-\beta)^{2}}{(1+\beta)^{2}} S^{2}(Z)\right] I_{A}(X) \\
& -\frac{1}{\sigma^{2}}\left[\frac{1}{(1-\beta)^{2}}+\frac{\lambda^{3}}{(1-\beta)^{3}} Z R(Z)+\frac{\lambda^{2}}{(1-\beta)^{2}} R^{2}(Z)\right] I_{A^{\prime}}(X) \\
& \frac{\partial^{2} l(\theta ; X)}{\partial \sigma \partial \mu}=-\frac{1}{\sigma^{2}}\left[\frac{2 Z}{(1+\beta)^{2}}-\frac{\lambda(1-\beta)}{1+\beta} S(Z)+\frac{\lambda^{3}(1-\beta)}{(1+\beta)^{3}} Z^{2} S(Z)+\frac{\lambda^{2}(1-\beta)^{2}}{(1+\beta)^{2}} Z S^{2}(Z)\right] I_{A}(X) \\
& -\frac{1}{\sigma^{2}}\left[\frac{2 Z}{(1-\beta)^{2}}-\frac{\lambda}{1-\beta} R(Z)+\frac{\lambda^{3}}{(1-\beta)^{3}} Z^{2} R(Z)+\frac{\lambda^{2}}{(1-\beta)^{2}} Z R^{2}(Z)\right] I_{A^{\prime}}(X), \\
& \frac{\partial^{2} l(\theta ; X)}{\partial \lambda \partial \mu}=-\frac{1}{\sigma}\left[\frac{1-\beta}{1+\beta} S(Z)-\frac{\lambda^{2}(1-\beta)}{(1+\beta)^{3}} Z^{2} S(Z)-\frac{\lambda(1-\beta)^{2}}{(1+\beta)^{2}} Z S^{2}(Z)\right] I_{A}(X) \\
& -\frac{1}{\sigma}\left[\frac{1}{1-\beta} R(Z)-\frac{\lambda^{2}}{(1-\beta)^{3}} Z^{2} R(Z)-\frac{\lambda}{(1-\beta)^{2}} Z R^{2}(Z)\right] I_{A^{\prime}}(X), \\
& \frac{\partial^{2} l(\theta ; X)}{\partial \beta \partial \mu}=-\frac{1}{\sigma}\left[\frac{2 Z}{(1+\beta)^{3}}-\frac{2 \lambda}{(1+\beta)^{2}} S(Z)-\frac{\lambda(1-\beta)}{(1+\beta)} S(Z) T(Z)+\frac{\lambda^{3}(1-\beta)}{(1+\beta)^{4}} Z^{2} S(Z)\right. \\
& \left.+\frac{\lambda^{2}(1-\beta)^{2}}{(1+\beta)^{3}} Z S^{2}(Z)\right] I_{A}(X) \\
& -\frac{1}{\sigma}\left[-\frac{2 Z}{(1-\beta)^{3}}+\frac{\lambda}{(1-\beta)^{2}} R(Z)-\frac{\lambda^{3}}{(1-\beta)^{4}} Z^{2} R(Z)-\frac{\lambda^{2}}{(1-\beta)^{3}} Z R^{2}(Z)\right] I_{A^{\prime}}(X), \\
& \frac{\partial^{2} l(\theta ; X)}{\partial \sigma^{2}}=\frac{1}{\sigma^{2}}-\frac{1}{\sigma^{2}}\left[\frac{3 Z^{2}}{(1+\beta)^{2}}-\frac{2 \lambda(1-\beta)}{1+\beta} Z S(Z)+\frac{\lambda^{3}(1-\beta)}{(1+\beta)^{3}} Z^{3} S(Z)\right. \\
& \left.+\frac{\lambda^{2}(1-\beta)^{2}}{(1+\beta)^{2}} Z^{2} S^{2}(Z)\right] I_{A}(X) \\
& -\frac{1}{\sigma^{2}}\left[\frac{3 Z^{2}}{(1-\beta)^{2}}-\frac{2 \lambda}{1-\beta} Z R(Z)+\frac{\lambda^{3}}{(1-\beta)^{3}} Z^{3} R(Z)+\frac{\lambda^{2}}{(1-\beta)^{2}} Z^{2} R^{2}(Z)\right] I_{A^{\prime}}(X), \\
& \frac{\partial^{2} l(\theta ; X)}{\partial \lambda \partial \sigma}=-\frac{1}{\sigma}\left[\frac{1-\beta}{1+\beta} Z S(Z)-\frac{\lambda^{2}(1-\beta)}{(1+\beta)^{3}} Z^{3} S(Z)-\frac{\lambda(1-\beta)^{2}}{(1+\beta)^{2}} Z^{2} S^{2}(Z)\right] I_{A}(X) \\
& -\frac{1}{\sigma}\left[\frac{1}{1-\beta} Z R(Z)-\frac{\lambda^{2}}{(1-\beta)^{3}} Z^{3} R(Z)-\frac{\lambda}{(1-\beta)^{2}} Z^{2} R^{2}(Z)\right] I_{A^{\prime}}(X) \text {, } \\
& \frac{\partial^{2} l(\theta ; X)}{\partial \beta \partial \sigma}=-\frac{1}{\sigma}\left[\frac{2 Z^{2}}{(1+\beta)^{3}}-\frac{2 \lambda}{(1+\beta)^{2}} Z S(Z)-\frac{\lambda(1-\beta)}{(1+\beta)} Z S(Z) T(Z)+\frac{\lambda^{3}(1-\beta)}{(1+\beta)^{4}} Z^{3} S(Z)\right. \\
& \left.+\frac{\lambda^{2}(1-\beta)^{2}}{(1+\beta)^{3}} Z^{2} S^{2}(Z)\right] I_{A}(X) \\
& -\frac{1}{\sigma}\left[-\frac{2 Z^{2}}{(1-\beta)^{3}}+\frac{\lambda}{(1-\beta)^{2}} Z R(Z)-\frac{\lambda^{3}}{(1-\beta)^{4}} Z^{3} R(Z)-\frac{\lambda^{2}}{(1-\beta)^{3}} Z^{2} R^{2}(Z)\right] I_{A^{\prime}}(X) \text {, }
\end{aligned}
$$




$$
\begin{aligned}
& \frac{\partial^{2} l(\theta ; X)}{\partial \lambda^{2}}=-\left[\frac{\lambda(1-\beta)}{(1+\beta)^{3}} Z^{3} S(Z)+\frac{(1-\beta)^{2}}{(1+\beta)^{2}} Z^{2} S^{2}(Z)\right] I_{A}(X) \\
& -\left[\frac{\lambda}{(1-\beta)^{3}} Z^{3} R(Z)+\frac{1}{(1-\beta)^{2}} Z^{2} R^{2}(Z)\right] I_{A^{\prime}}(X), \\
& \frac{\partial^{2} l(\theta ; X)}{\partial \beta \partial \lambda}=-\left[\frac{1-\beta}{1+\beta} Z S(Z) T(Z)+\frac{2}{(1+\beta)^{2}} Z S(Z)-\frac{\lambda^{2}(1-\beta)}{(1+\beta)^{4}} Z^{3} S(Z)\right. \\
& \left.-\frac{\lambda(1-\beta)^{2}}{(1+\beta)^{3}} Z^{2} S^{2}(Z)\right] I_{A}(X) \\
& -\left[-\frac{1}{(1-\beta)^{2}} Z R(Z)+\frac{\lambda^{2}}{(1-\beta)^{4}} Z^{3} R(Z)+\frac{\lambda}{(1-\beta)^{3}} Z^{2} R^{2}(Z)\right] I_{A^{\prime}}(X),
\end{aligned}
$$

and

$$
\begin{aligned}
& \frac{\partial^{2} l(\theta ; X)}{\partial \beta^{2}}=-\left[-\frac{1}{(1+\beta)^{2}}+\frac{3 Z^{2}}{(1+\beta)^{4}}-\frac{4 \lambda}{(1+\beta)^{3}} Z S(Z)+\frac{\lambda^{3}(1-\beta)}{(1+\beta)^{4}} Z^{3} S(Z)\right. \\
& \left.+\frac{\lambda^{2}(1-\beta)^{2}}{(1+\beta)^{3}} Z^{2} S^{2}(Z)-\frac{\lambda(1-\beta)(2+\beta)}{(1+\beta)^{2}} Z S(Z) T(Z)+T^{2}(Z)\right] I_{A}(X) \\
& -\left[\frac{3 Z^{2}}{(1-\beta)^{4}}-\frac{2 \lambda}{(1-\beta)^{3}} Z R(Z)+\frac{\lambda^{3}}{(1-\beta)^{5}} Z^{3} R(Z)+\frac{\lambda^{2}}{(1-\beta)^{4}} Z^{2} R^{2}(Z)\right] I_{A^{\prime}}(X) .
\end{aligned}
$$

For the derivation of the Fisher information of (1.1), the following integrals need to be calculated, which can be done numerically by using the software R [6]:

$$
\begin{aligned}
& \rho_{k}=\rho_{k}(\lambda, \beta)=\frac{2(-1)^{k}}{1+\beta} \int_{0}^{\infty} \frac{t^{k} \phi^{2}\left(\frac{\lambda t}{1+\beta}\right)}{1-(1-\beta) \Phi\left(\frac{\lambda t}{1+\beta}\right)} \phi\left(\frac{t}{1+\beta}\right) d t, \\
& \eta_{k}=\eta_{k}(\lambda, \beta)=2 \int_{0}^{\infty} \frac{t^{k} \phi^{2}\left(\frac{\lambda t}{1-\beta}\right)}{\Phi\left(\frac{\lambda t}{1-\beta}\right)} \phi\left(\frac{t}{1-\beta}\right) d t \\
& \kappa_{k}=\kappa_{k}(\lambda, \beta)=\frac{2(-1)^{k}}{1+\beta} \int_{0}^{\infty} \frac{t^{k} \phi\left(\frac{\lambda t}{1+\beta}\right)}{1-(1-\beta) \Phi\left(\frac{\lambda t}{1+\beta}\right)} \phi\left(\frac{t}{1+\beta}\right) \Phi\left(\frac{\lambda t}{1+\beta}\right) d t,
\end{aligned}
$$

and

$$
v_{k}=v_{k}(\lambda, \beta)=\frac{2(-1)^{k}}{1+\beta} \int_{0}^{\infty} \frac{t^{k} \Phi^{2}\left(\frac{\lambda t}{1+\beta}\right)}{1-(1-\beta) \Phi\left(\frac{\lambda t}{1+\beta}\right)} \phi\left(\frac{t}{1+\beta}\right) d t
$$

where $k=0,1,2$. Define also, $\rho_{k}^{*}=\rho_{k}(\lambda, 0), \eta_{k}^{*}=\eta_{k}(\lambda, 0), \kappa_{k}^{*}=\kappa_{k}(\lambda, 0) \mathrm{y}$ $v_{k}^{*}=v_{k}(\lambda, 0)$. 


\title{
References
}

[1] Azzalini, A. A class of distributions which includes the normal ones. Scandinavian Journal of Statistics. 12, pp. 171-178, (1985).

[2] Chiogna, M. A note on the asymptotic distribution of the maximum likelihood estimator for the scalar skew-normal distribution. Statistical Methods \& Applications. 14, pp. 331-341, (2005).

[3] DiCiccio, T. J. and Monti, A. C. Inferential Aspects of the Skew Exponential Power Distribution. Journal of the American Statistical Association. 99(466), pp. 439-450, (2004).

[4] Gómez, H. W., Salinas, H. S. and Bolfarine, H., Generalized skewnormal models: Properties and inference. Statistics: A Journal of Theoretical and Applied Statistics. 40(6), pp. 495-505, (2006).

[5] Lehman, E. L. \& Casella, G. Theory of Point Estimation (2nd edition). New York, NY: Springer, (1998).

[6] R Development Core Team, R : A language and environment for statistical computing. R Foundation for Statistical Computing, Vienna, Austria. ISBN 3-900051-07-0, URL http://www.R-project.org, (2010).

[7] Rotnitzky, A. Cox, D. R., Bottai, M. and Robins, J., Likelihood-based inference with singular information matrix. Bernoulli. 6, pp. 243-284, (2000).

\author{
Héctor W. Gómez \\ Departamento de Matemáticas \\ Facultad de Ciencias Básicas \\ Universidad de Antofagasta \\ Chile \\ e-mail: hgomez@uantof.cl \\ and
}


Hugo S. Salinas

Departamento de Matemáticas

Facultad de Ingeniería

Universidad de Atacama

Chile

e-mail: hsalinas@mat.uda.cl 